\title{
Accuracy of Multidetector Computed Tomography for N Staging of Gastric Cancer in Eastern Asia: A Meta-analysis and Systematic Review
}

Lei Xu

Nanjing Medical University Second Affiliated Hospital https://orcid.org/0000-0002-7092-8280

Xiang Gao

Nanjing Medical University Second Affiliated Hospital

Jianping Zhang ( $\nabla$ drzhangjp@njmu.edu.cn )

https://orcid.org/0000-0002-1929-792X

Research article

Keywords: Gastric cancer, multidetector computed tomography, lymph node metastasis, $\mathrm{N}$ staging, Eastern Asia

Posted Date: July 9th, 2020

DOl: https://doi.org/10.21203/rs.3.rs-38420/v1

License: (c) (i) This work is licensed under a Creative Commons Attribution 4.0 International License. Read Full License 


\section{Abstract}

Background:Eastern Asia is a region with a high incidence of gastric cancer (GC), and the detection rate of early gastric cancer (EGC) has been rising in recent decades. ${ }^{2}$ With the development of endoscopic submucosal dissection (ESD) and the scope of its indications gradually expanded,the treatment for EGC is becoming a hot topic, which involves accurate preoperative evaluation. ${ }^{3}$ The purpose of this study was to assess overall accuracy of multidetector computed tomography (MDCT) in N staging of GC in Eastern Asia. $^{4}$

Methods: A comprehensive search was conducted to collect all relevant published studies about MDCT in assessing N staging of GC in Eastern Asia, within the PubMed, Cochrane library and Web of science databases from January 01,2002 to April $01,2020 .{ }^{5}$ The raw data from the included studies calculated the pooled sensitivity, specificity, positive predictive value (PPV), and negative predictive value (NPV) for evaluating the performance of MDCT. ${ }^{6}$ The studies were grouped according to different criteria to assess the effect of possible factors on the sensitivity of MDCT. ${ }^{7}$

Results: fourteen studies (5119 patients) were included in this analysis. ${ }^{8}$ In Eastern Asia, MDCT had a pooled accuracy of $67 \%$ (95\% confidence interval [Cl] 0.62-0.72, I $=90.1 \%$ ) for $\mathrm{N}$ staging. By subgroup analysis, reference diameter of lymph node metastasis (LNM) and degree of tumor invasion (DTI) were correlated with the sensitivity of $\mathrm{N}$ staging. ${ }^{9}$ And the pooled sensitivity for $\mathrm{N}+$ of EGC was relatively low. ${ }^{10}$

Conclusions: In Eastern Asia, as the influence of reference diameter of LNM and DTI, the sensitivity of MDCT in N staging of GC was insufficient. ${ }^{11}$ This may lead to incorrect preoperative staging, so that patients with GC may be over treated or inadequate lymph node (LN)dissection. ${ }^{12}$ The hierarchical assessment strategy may significantly improve the sensitivity for N+ of EGC to make appropriate methods of treatment. ${ }^{13}$

\section{Background}

Gastric cancer (GC) is the fifth most frequently diagnosed cancer and the third leading cause of cancer death worldwide [1]. ${ }^{14} \mathrm{GC}$ causes a particularly high proportion of cancer deaths in China and it has been the most common malignancy in Japan $[2,3] .{ }^{15}$ It was estimated that Eastern Asia had the highest mortality rate and the incidence of GC in the world in 2008 was about 1 million, half of which occurred in Eastern Asia. ${ }^{16}$ The detection rate of early gastric cancer (EGC) has been gradually increasing in recent decades, which may be related to the popularization of gastroscopy screening and the improvement of people's health concept. ${ }^{17}$ Therefore, endoscopic submucosal dissection (ESD) has gradually entered the middle of people's eyes and is committed to removing EGC within the scope of indications. ${ }^{18}$ Compared with laparoscopic and laparotomy, ESD has many advantages, such as less trauma and faster recovery, but ESD cannot effectively remove positive lymph nodes (LNs). ${ }^{19}$ The indication of ESD is mainly GC T1a staging without lymph node metastasis $(\mathrm{LNM}) .{ }^{20}$ Through clinical comparative tests, the reliability and 
safety of ESD's extended indication has been verified [4]. ${ }^{21}$ For patients who have been treated with ESD, based on the potential risk of residual tumors in the stomach and / or LNM, gastrectomy + LN dissection was performed. ${ }^{22}$ The actual incidence of LNM in patients with additional gastrectomy for incomplete tumor removal is about $5 \%-10 \%[5-8] .{ }^{23}$ This means that the vast majority of patients were overtreated. ${ }^{24}$ Therefore, accurate preoperative staging of GC, especially N staging of EGC, is essential for the selection of appropriate treatment. ${ }^{25} \mathrm{~A}$ variety of tests have been used to assess $\mathrm{LN}$ status in GC such as multidetector computed tomography (MDCT), endoscopic ultrasonography (EUS), magnetic resonance imaging (MRI), and positron emission tomography/computed tomography (PET/CT) [9]. ${ }^{26} \mathrm{MDCT}$ has been used as a hot tool for continuous research to more accurately assess the preoperative staging of GC since its invention $[10,11] .{ }^{27}$ With the development of the times and technological innovation, MDCT has been greatly improved and is currently considered as one of the most reliable preoperative staging techniques for GC [12-15]. ${ }^{28}$ When using MDCT alone, its accuracy has been reported to reach $89 \%$ [16, 17]. ${ }^{29}$ There were also many conclusions about the accuracy of MDCT in evaluating the $\mathrm{N}$ staging of GC. ${ }^{30}$ Fujikawa et al. [18] showed that the main method for preoperative evaluation of $\mathrm{N}$ staging of GC with MDCT was to detect the size of LN diameter, but the relatively low sensitivity of MDCT led to nonnegligible false negative results. ${ }^{31}$ Cimavilla et al. [19] concluded that the pooled sensitivity and accuracy of MDCT for $\mathrm{N}$ staging were $55 \%$ and $64 \%$, respectively, through the comparison between MDCT and EUS. ${ }^{32}$ However, few studies have studied the accuracy of MDCT in N staging of GC in Eastern Asia with high morbidity and mortality. ${ }^{33}$ The purpose of this study was to conduct a systematic review and metaanalysis of studies on the diagnostic accuracy of MDCT in the evaluation of LNM of GC in Eastern Asia. $^{34}$

\section{Methods}

Search strategy: Two authors searched systematically from the PubMed, Cochrane library and Web of science databases for all relevant articles published from January 01, 2002 to April 01, 2020, that about MDCT for preoperative N staging of GC in Eastern Asia. ${ }^{35}$ The following keywords were used in the search article: "(stomach neoplasm) OR (gastric neoplasm) OR (stomach cancers) OR (gastric cancer) OR (cancer, gastric) OR (gastric cancers) OR (stomach cancer)" and "(lymph node metastasis) OR (lymph node involvement) OR (lymph node staging) OR (lymphatic metastasis) OR (nodal involvement) OR (lymph node status) OR (N staging)" and "(computed tomography) OR (multidetector computed tomography) OR (CT) OR (MDCT)". ${ }^{36}$ No language restrictions, and also manually checked whether the reference list of the related studies met the inclusion criteria. ${ }^{37}$

Study inclusion and exclusion criteria: inclusion criteria:(1) Number of samples $n \geq 30$ cases, no radiotherapy and chemotherapy before surgery, postoperative pathology confirmed GC; (2) Histopathological results after gastrectomy as the criterion for the evaluation of LNM; (3) Only studies contain original data that the sensitivity specificity, positive predictive value (PPV), and negative predictive value (NPV) of N0, N1, N2 and N3 can be obtained or calculated respectively; (4) only studies in 
eastern Asian population. ${ }^{38}$ exclusion criteria:(1) Animal or in vitro studies;(2) Review articles, case reports, abstracts, meta-analyses, correspondence and meeting records. ${ }^{39}$

\section{Data Extraction, Synthesis and Analysis}

If the two authors encounter dispute in the inclusion or exclusion of articles, the dispute shall be settled through consultation with the third author. ${ }^{40}$ If no agreement can be reached, the article shall be removed to achieve the quality control of the included articles. ${ }^{41}$ The sensitivity, specificity, PPV and NPV of each N stage were calculated respectively from the raw data or known results with the bivariate random effects model. ${ }^{42}$ In addition, we divided studies into groups by reference diameter of LNM and the proportion of degree of tumor invasion (DTI) to evaluate possible factors on the accuracy of MDCT. ${ }^{43} \mathrm{P}<0.05$ or $1^{2}>50 \%$ indicated heterogeneity in statistical results. ${ }^{44}$ Endnote- $x 9$, noteexpress-3.2, and statase-64 were used to organize articles, process data, and make graphics. ${ }^{45}$

\section{Results}

A systematic search was conducted to initially identify 4333 articles for the first review. ${ }^{46}$ After deleting duplicates and screening related titles and abstracts, the remaining 233 articles were reviewed for full text, and a total of 14 studies [20-33] were finally adopted in accordance with the inclusion criteria

(Fig. 1). ${ }^{47}$ The relevant characteristics of these studies are listed in table $1 .{ }^{48} \mathrm{~A}$ total of 5119 patients with GC in Eastern Asia received detailed staging of MDCT before surgery. ${ }^{49}$

\section{N staging}

The overall diagnostic accuracy of MDCT for $\mathrm{N}$ staging varied between $43 \%$ and $86.3 \%$, and the pooled accuracy was $67 \%(95 \% \mathrm{Cl} 0.62-0.72, \mathrm{I}=90.1 \%, \mathrm{P}<0.05)$ in Eastern Asia. ${ }^{50}$ The range of individual study sensitivity, specificity, PPV, NPV are presented in Table 2 , the results of each study vary greatly for $\mathrm{N}+.^{51}$ For N0, the pooled sensitivity, specificity, PPV and NPV were $0.77(95 \% \mathrm{Cl} 0.69-0.84), 0.77(0.67-0.86)$, $0.75(0.71-0.78)$, and $0.82(0.78-0.86)$ respectively (Fig. 2) ${ }^{52}$ For N1, the results were $0.55(0.43-0.66)$, $0.83(0.78-0.88), 0.47(0.38-0.56)$ and $0.86(0.84-0.89)$ respectively (Fig. 3). ${ }^{53}$ The pooled sensitivity, specificity, PPV and NPV of MDCT for N2 were $0.43(0.35-0.52), 0.9(0.85-0.94), 0.51(0.38-0.63)$ and 0.89 (0.86-0.91) respectively (Fig. 4), and were $0.42(0.31-0.53), 0.99(0.98-1.0), 0.56(0.34-0.77)$ and $0.92(0.88-0.96)$ for N3 respectively (Fig. 5). ${ }^{54}$ The calculated $\mathrm{I}^{2}$ value for above results were almost all > $50 \%$ and the $P$ value was often $<0.05$, indicated that there was considerable heterogeneity among the included studies. ${ }^{55}$ In terms of grouping, the pooled sensitivity for NO and N + were 0.66 and 0.88 in 6 studies $[20,22,26,28,32,33]$ which the reference diameter of LNM in MDCT was $<8 \mathrm{~mm}$, and another group $[21,23,25,27,30,31]$ of the reference was $\geq 8 \mathrm{~mm}$, the pooled sensitivity for $\mathrm{N} 0$ and $\mathrm{N}+$ were 0.91 and 0.52 respectively. ${ }^{56}$ The result may instruct that appropriate reduction of the reference diameter of LNM could increase MDCT sensitivity for LNM in Eastern Asia. ${ }^{57}$ The other grouping was about DTI, which was divided into two groups according to the proportion of T3 and T4 in T stage by pathological 
results, $<50 \%$ (the majority of patients with $\operatorname{EGC}$ ) $[23,25,27,31]$ or $\geq 50 \%$ (the majority of patients with advanced gastric cancer(AGC)) $[21,24,26,28-30] .{ }^{58}$ The pooled sensitivity for $\mathrm{N} O$ and $\mathrm{N}+$ were 0.92 and 0.49 in the proportion $<50 \%$, and were 0.6 and 0.84 in the proportion $\geq 50 \%$ respectively. ${ }^{59}$ It was possible to confirm that MDCT staging sensitivity significantly varied in EGC and AGC in Eastern Asia ${ }^{60}$ For $\mathrm{N}$ +, there may be better sensitivity for preoperative diagnosis of LNM of AGC by MDCT. ${ }^{61}$

Table 1. Characteristics of included studies

\begin{tabular}{|c|c|c|c|c|c|c|c|}
\hline Author & Published year & County & Sam ple Size & overall accuracy & Reference diem eter of LNM & Proportion of PT3+PT4 & Enhanced scanning \\
\hline W uetal & 2008 & China & 56 & 0.43 & $5 \mathrm{~m} \mathrm{~m}$ & $/$ & Yes \\
\hline W ang etal & 2009 & China & 33 & 0.76 & $10 \mathrm{~m} \mathrm{~m}$ & $62 \%$ & Yes \\
\hline Jie etal & 2009 & China & 118 & 0.68 & $5 \mathrm{~m} \mathrm{~m}$ & / & Yes \\
\hline $\mathrm{H}$ wang etal & 2010 & Korea & 247 & 0.63 & $8 \mathrm{~m} \mathrm{~m}$ & $9 \%$ & Yes \\
\hline Fang etal & 2012 & China & 126 & 0.75 & / & $65 \%$ & Yes \\
\hline Hasegawa etal & 2013 & Japan & 315 & 0.76 & $8 \mathrm{~m} \mathrm{~m}$ & $21 \%$ & Yes \\
\hline Feng etal & 2013 & China & 610 & 0.67 & $6 \mathrm{~m} \mathrm{~m}$ & $81 \%$ & Yes \\
\hline $\mathrm{Km}$ etal & 2013 & Korea & 171 & 0.78 & $8 \mathrm{~m} \mathrm{~m}$ & $40 \%$ & Yes \\
\hline Cao etal & 2014 & China & 185 & 0.69 & $6 \mathrm{~mm}$ & $74 \%$ & Yes \\
\hline Yong etal & 2014 & China & 194 & 0.56 & / & $72 \%$ & Yes \\
\hline O hashietal & 2016 & Japan & 218 & 0.46 & $8 \mathrm{~m} \mathrm{~m}$ & $72 \%$ & Yes \\
\hline Bando etal & 2017 & Japan & 2699 & 0.64 & $10 \mathrm{~m} \mathrm{~m}$ & $38 \%$ & Yes \\
\hline M eietal & 2019 & China & 56 & 0.7 & $6 \mathrm{~m} \mathrm{~m}$ & / & Yes \\
\hline Jieng etal & 2019 & China & 91 & 0.86 & $5 \mathrm{~m} \mathrm{~m}$ & / & Yes \\
\hline
\end{tabular}

Table2. The range of individual study sensitivity, specificity, PPV,NPV.

\begin{tabular}{|lllll|}
\hline N staging & sensitivity & specificity & PPV & NPV \\
\hline N0 & $0.5-0.97$ & $0.45-0.99$ & $0.6-0.93$ & $0.6-0.98$ \\
\hline N1 & $0.18-0.88$ & $0.68-1.0$ & $0.16-1.0$ & $0.73-0.99$ \\
\hline N2 & $0.14-0.74$ & $0.72-0.99$ & $0.14-0.8$ & $0.79-0.94$ \\
\hline N3 & $0-1.0$ & $0.9-1.0$ & $0-1.0$ & $0.84-1.0$ \\
\hline
\end{tabular}

\section{Discussion}

This study evaluated data from multiple studies (number of patients $n=5119$ ) to assess the diagnostic performance of MDCT in N staging of GC in Eastern Asia. ${ }^{62}$ Meta-analytical statistical methods were used to integrate and subgroup the data and it was found that the preoperative diagnostic performance of MDCT was not very reliable and the pooled accuracy of $\mathrm{N}$ staging was only $67 \%(95 \% \mathrm{Cl} 0.62-0.72) .{ }^{63}$ And the sensitivity of MDCT in the clinical diagnosis of GC (especially EGC) N staging was not satisfactory. ${ }^{64}$

The data were grouped according to the reference diameter of LNM and the proportion of DTI. ${ }^{65}$ The subgroup analysis results indicated that when the reference diameter of LNM was $<8 \mathrm{~mm}$ or $\geq 8 \mathrm{~mm}$, the sensitivity of MDCT for $\mathrm{N}+$ staging was significantly different, and the sensitivity of the former was 
significantly greater than that of the latter. ${ }^{66}$ Preoperative LNM can be determined from the size of the LN, morphology, strengthening methods to comprehensive analysis. ${ }^{67}$ The American Joint Committee for Cancer (AJCC) and the Japanese Gastric Cancer Association (JGCA) are currently widely recognized TNM staging criteria for GC. ${ }^{68}$ However, the above two staging systems did not provide a reference standard for the preoperative determination of positive LNs by MDCT. ${ }^{69}$ Therefore, the preoperative diagnosis of LNM in GC has always been the focus of research. ${ }^{70}$ Generally, the diameter of LN is $\geq 8 \mathrm{~mm}$, and LNs are considered to be involved. ${ }^{71}$ For the grouping of DTI, MDCT in patients with mostly AGC was more sensitive for $\mathrm{N}+$ staging than that in patients with mostly EGC, suggesting that the proportion of DTI was an important variable affecting the sensitivity, while MDCT was poor in determining $\mathrm{N}$ staging with EGC. ${ }^{72}$ In AGC with LNM, large or clustered LNs are usually found around the primary lesion [34]. ${ }^{73}$ However, EGC with few LNM usually has very small metastatic LNs and is less typical in MDCT imaging than in AGC [35]. ${ }^{74}$ Microscopic metastases found in the LNs of patients with EGC are often classed as negative because the diameters do not meet the criteria for LNM, which makes accurate $\mathrm{N}$ staging more difficult in EGC than in AGC [12]. ${ }^{75}$ This means that MDCT is challenging in the proper clinical evaluation of $\mathrm{LN}$ involvement in EGC. ${ }^{76}$ Early reports showed that the accuracy of preoperative $\mathrm{N}$ staging for GC ranged from $51-76 \%$, and the sensitivity of $\mathrm{N}$ staging ranged from $48-91 \%$ [12]. ${ }^{77}$ Fukuya et al. [36] took $>5 \mathrm{~mm}$ as the standard, with $\mathrm{N}$ staging sensitivity of $75 \%$ and specificity of $42 \% .{ }^{78} \mathrm{D}$ 'elia et al. [37] took the shorter diameter of perigastric $\mathrm{LN}>6 \mathrm{~mm}$ and the shorter diameter of peripheral $\mathrm{LN}>8 \mathrm{~mm}$ as the standard, and the sensitivity and specificity of $\mathrm{N}$ staging were $97.2 \%$ and $65.7 \%$, respectively. ${ }^{79}$ Research reports from different periods have shown that due to the effect of DTI on LN status not considered and the lack of reliable preoperative reference diameter criteria for the determination of positive LNs, the results fluctuated widely. ${ }^{80}$ Therefore, whether in Eastern Asia or elsewhere, in the preoperative evaluation of GC with MDCT, we need to establish more refined and specific criteria for LNM to detect LN status under different conditions. ${ }^{81}$

The effect of LNs at different locations on the accuracy of MDCT was also widely discussed. ${ }^{82}$ Pan et al. [38] showed that the results of LNs evaluation by MDCT at different sites showed different sensitivities compared with those of pathology, in addition, among the various metastatic LNs, MDCT showed better sensitivity in the splenic hilum, splenic artery and the sites with less curvature. ${ }^{83}$ The results of Jia et al. [22] indicated that the examination of LNs on CT depended on the contrast of surrounding structures, and clear anatomical structure, close to the large blood vessels, more fat in the abdominal cavity and LNs in the stomach area were easier to detect. ${ }^{84}$ In their study, group location diagnosis was adopted. ${ }^{85}$ For MDCT, according to the fifth edition of the AJCC TNM staging, the sensitivity of LNs in groups 1, 3, 7-9, and 11 was high, and the sensitivity of LNs in groups 4 and 5 was poor. ${ }^{86}$ Therefore, the lack of one-toone correspondence between the LNs evaluated by MDCT before operation and those detected by pathology after operation may make the accuracy of MDCT unreliable. ${ }^{87}$

As mentioned above, the existing accuracy of MDCT is not sufficient for the preoperative evaluation of EGC. ${ }^{88}$ In order to select appropriate surgical methods (ESD, gastrectomy + perigastric LN resection or 
enlarged resection) for patients to achieve precision medicine, hierarchical assessment may be effective according to the result of the subgroup analysis. ${ }^{89}$ In order to achieve the hierarchical assessment and standard development, imaging technicians should first assess the T staging of GC, and then stratify the patients according to the T staging: $\mathrm{EGC}(\mathrm{T} 1, \mathrm{~T} 2)$ with $<8 \mathrm{~mm}$ (such as $6 \mathrm{~mm})$ as the reference diameter of LNM, or AGC $(T 3, T 4)$ with $\geq 8 \mathrm{~mm}$ (such as $8 \mathrm{~mm}$ or $10 \mathrm{~mm}$ ) as the reference diameter of LNM. This hierarchical assessment can take into account both the effect of DTI on LNs and the effect of reference diameter of LNM on the sensitivity. ${ }^{90}$ Such a diagnostic process may greatly improve the accuracy of preoperative staging of EGC, avoid patients from being overtreated or timely adjust the surgical method to expand the scope of surgery. ${ }^{91}$ About the LNs in different locations, a criterion for clinical diagnosis of $\mathrm{N}$ stages is needed. ${ }^{92}$ MDCT in the preoperative assessment of LNs status should be accurate to mark specific location of the suspected LNs, surgeons should accurately remove and mark the corresponding LNs to achieve a one-to-one relationship with the pathology. ${ }^{93}$ This not only enables a real preoperative evaluation of $\mathrm{N}$ staging with MDCT, but also enables a more accurate understanding of the biological behavior of LNM in GC. ${ }^{94}$

\section{Conclusions}

Through the above presentation and discussion of the results, this study indicated that the overall accuracy of MDCT for $\mathrm{N}$ staging of GC is relatively low in Eastern Asia, especially the sensitivity for $\mathrm{N}+$ of EGC, and its sensitivity may be related to the reference diameter of LNM, DTI and the location of LN. ${ }^{95}$ In order to address the effect of LN differences between EGC and AGC on MDCT, a concept of hierarchical assessment was proposed, which may greatly improve the accuracy of MDCT in the diagnosis of preoperative N staging of EGC to select appropriate surgical procedures for patients. ${ }^{96}$

\section{Abbreviations}

GC

gastric cancer

EGC

early gastric cancer

ESD

endoscopic submucosal dissection

LN

lymph node

LNM

lymph node metastasis

MDCT

multidetector computed tomography

EUS

endoscopic ultrasonography 
MRI

magnetic resonance imaging

PET/CT

positron emission tomography/computed tomography

PPV

positive predictive value

NPV

negative predictive value

DTI

degree of tumor invasion

AGC

advanced gastric cancer

AJCC

the American Joint Committee for Cancer

JGCA

the Japanese Gastric Cancer Association

\section{Declarations}

\section{Ethics approval and consent to participate:}

Our study did not require an ethical board approval because the included studies were published articles and there are no experiments involving humans or animals.

Consent for publication:

not applicable.

Availability of data and materials:

The datasets generated during and/or analysed during the current study are available in the [NCBIPubMed] for English articles,

\section{Competing interests:}

no potential conflict of interests.

\section{Funding:}

not applicable. 


\section{Authors' contributions:}

$\mathrm{XL}$ and $\mathrm{GX}$ searched and screened the articles from the database, $\mathrm{XL}$ and ZJP reviewed and confirmed the included articles, and XL wrote and modified the article. All authors' read and approved the final manuscript.

\section{Acknowledgements:}

Thanks to the surgeons of the Second Affiliated Hospital of Nanjing Medical University for their comments on this article.

\section{References}

1. Bray F, Ferlay J, Soerjomataram I, et al. Global cancer statistics 2018: GLOBOCAN estimates of incidence and mortality worldwide for 36 cancers in 185 countries. CA Cancer J Clin. 2018;68:394424.

2. Chen W, Zheng R, Baade PD, et al. Cancer statistics in China, 2015. CA Cancer J Clin. 2016;66:11532.

3. Nomura K. Cancer statistics in Japan, 2005. Tokyo: Foundation for Promotion Cancer Research; 2006.

4. Kim HJ, Kim SG, Kim J, et al. Clinical outcomes of early gastric cancer with non-curative resection after pathological evaluation based on the expanded criteria. PLOS ONE. 2019;14:e224614.

5. Ryu KW, Choi IJ, Doh YW, et al. Surgical indication for non-curative endoscopic resection in early gastric cancer. Ann Surg Oncol. 2007;14:3428-34.

6. Kim ER, Lee H, Min BH, et al. Effect of rescue surgery after non-curative endoscopic resection of early gastric cancer. Br J Surg. 2015;102:1394-401.

7. ang Y, Kim HJ, Lim SG. JH, et al. Predictors of lymph node metastasis in patients with non-curative endoscopic resection of early gastric cancer. Surg Endosc. 2015;29:1145-55.

8. Suzuki H, Oda I, Abe S, et al. Clinical outcomes of early gastric cancer patients after noncurative endoscopic submucosal dissection in a large consecutive patient series. Gastric Cancer. 2017;20:679-89.

9. Hwang SW, Lee DH. Is endoscopic ultrasonography still the modality of choice in preoperative staging of gastric cancer? World J Gastroenterol. 2014;20:13775-82.

10. Davies J, Chalmers AG, Sue-Ling HM, et al. Spiral computed tomography and operative staging of gastric carcinoma: a comparison with histopathological staging. GUT. 1997;41:314-9.

11. Habermann CR, Weiss F, Riecken R, et al. Preoperative staging of gastric adenocarcinoma: comparison of helical CT and endoscopic US. RADIOLOGY. 2004;230:465-71. 
12. Kim AY, Kim HJ, Ha HK. Gastric cancer by multidetector row CT: preoperative staging. ABDOM IMAGING. 2005;30:465-72.

13. Kim HJ, Kim AY, Oh ST, et al. Gastric cancer staging at multi-detector row CT gastrography: comparison of transverse and volumetric CT scanning. RADIOLOGY. 2005;236:879-85.

14. Chen CY, Hsu JS, Wu DC, et al. Gastric cancer: preoperative local staging with 3D multi-detector row CT-correlation with surgical and histopathologic results. RADIOLOGY. 2007;242:472-82.

15. Kwee RM, Kwee TC. Imaging in assessing lymph node status in gastric cancer. GASTRIC CANCER. 2009;12:6-22.

16. Yang DM, Kim HC, Jin W, et al. 64 multidetector-row computed tomography for preoperative evaluation of gastric cancer: histological correlation. J Comput Assist Tomogr. 2007;31:98-103.

17. Shimizu K, Ito K, Matsunaga N, Shimizu A, Kawakami Y. Diagnosis of gastric cancer with MDCT using the water-filling method and multiplanar reconstruction: CT-histologic correlation. AJR Am J Roentgenol. 2005;185:1152-8.

18. Fujikawa $\mathrm{H}$, Yoshikawa $\mathrm{T}$, Hasegawa $\mathrm{S}$, et al. Diagnostic value of computed tomography for staging of clinical T1 gastric cancer. Ann Surg Oncol. 2014;21:3002-7.

19. Cimavilla Roman M, de la Serna Higuera C, Loza Vargas LA, et al. Endoscopic ultrasound versus multidetector computed tomography in preoperative gastric cancer staging. Rev esp de enfermedades digestivas. 2017;109:761-7.

20. Wu B, Lu T, Chen XZ, et al. Value of 64-row Helical CT in Detection and Staging of Metastatic Lymphadenopathy of Gastric Cancer. Journal of Clinical Radiology. 2008;27:1060-4.

21. Wang G, Cheng X, Wang ZM, et al. Diagnostic Value of MSCT with Two Oral Contrast Agents Filling Method in TNM Staging of Gastric Carcinoma. Chinese Journal of Clinical Oncology. 2009;22:126670.

22. Jia HP, Zhang H, Pan ZL, et al. Preoperative MDCT Evaluation of Gastric Cancer for N -staging. Chinese Computed Medical Imaging. 2009;15:338-44.

23. Hwang SW, Lee DH, Lee SH, et al. Preoperative staging of gastric cancer by endoscopic ultrasonography and multidetector-row computed tomography. J Gastroenterol Hepatol. 2010;25:512-8.

24. Fang WL, Peng HX, Li J, et al. Clinical analysis of endoscopic ultrasonography in preoperative TNM staging of 126 gastric cancer cases. Chinese Journal of Digestion. 2012;32:731-4.

25. Hasegawa S, Yoshikawa T, Shirai J, et al. A prospective validation study to diagnose serosal invasion and nodal metastases of gastric cancer by multidetector-row CT. ANN SURG ONCOL. 2013;20:201622.

26. Feng XY, Wang W, Luo GY, et al. Comparison of endoscopic ultrasonography and multislice spiral computed tomography for the preoperative staging of gastric cancer - results of a single institution study of 610 Chinese patients. PLoS One. 2013;8:e78846. 
27. Kim SH, Kim JJ, Lee JS, et al. Preoperative N staging of gastric cancer by stomach protocol computed tomography. J GASTRIC CANCER. 2013;13:149-56.

28. Cao L, Shan XH, Wang YF, et al. Comparison of diagnostic value in different parts and staging gastric cancer with 64-slice spiral CT. Journal of Practical Radiology. 2014;30:799-803.

29. Wang Y, Wang J, Hong Q. [Joint application of computed tomography and laparoscopy in preoperative staging of gastric cancer]. Zhonghua Yi Xue Za Zhi. 2014;94:769-71.

30. Ohashi M, Morita S, Fukagawa T, et al. Evaluation of 64-Channel Contrast-Enhanced Multi-detector Row Computed Tomography for Preoperative N Staging in cT2-4 Gastric Carcinoma. WORLD J SURG. 2016;40:165-71.

31. Bando $E$, Makuuchi R, Tokunaga $M$, et al. Impact of clinical tumor-node-metastasis staging on survival in gastric carcinoma patients receiving surgery. GASTRIC CANCER. 2017;20:448-56.

32. Mei JX, Wang S, Luo Y, et al ANALYSIS OF APPLICATION VALUE OF 64-SLICE ENHANCED CT IN PREOPERATIVE N STAGING OF GASTRIC CANCER. Acta Medica Mediterranea. 2019;35:595-601.

33. Jiang $M$, Wang $X$, Shan $X$, et al. Value of multi-slice spiral computed tomography in the diagnosis of metastatic lymph nodes and N-stage of gastric cancer. J INT MED RES. 2019;47:281-92.

34. Kawaguchi T, Ichikawa D, Komatsu S, et al. Impact of combination criteria of nodal counts and sizes on preoperative MDCT in advanced gastric cancer. World J Surg. 2016;40:158-64.

35. NakagawaM, Choi YY, An JY, et al. Difficulty of predicting the presence of lymph node metastases in patients with clinical early stage gastric cancer: a case control study. BMC Cancer. 2015;15:943.

36. Fukuya T, Honda H, Hayashi T. et a1.Lymph-node metastases: efficacy for detection with helical CT in patients with gastric cancer. Radiology. 1995;197:705-11.

37. D’Elia F, Zingarelli A, Palli D. et a1. Hydro-dynamic CT preoperative staging of gastric cancer: correlation with pathological findings. A prospective study of 107 cases. Eur Radiol. 2000;10:187785.

38. Pan Z, Zhang H, Yan C, et al. Determining gastric cancer resectability by dynamic MDCT. EUR RADIOL. 2010;20:613-20.

\section{Figures}




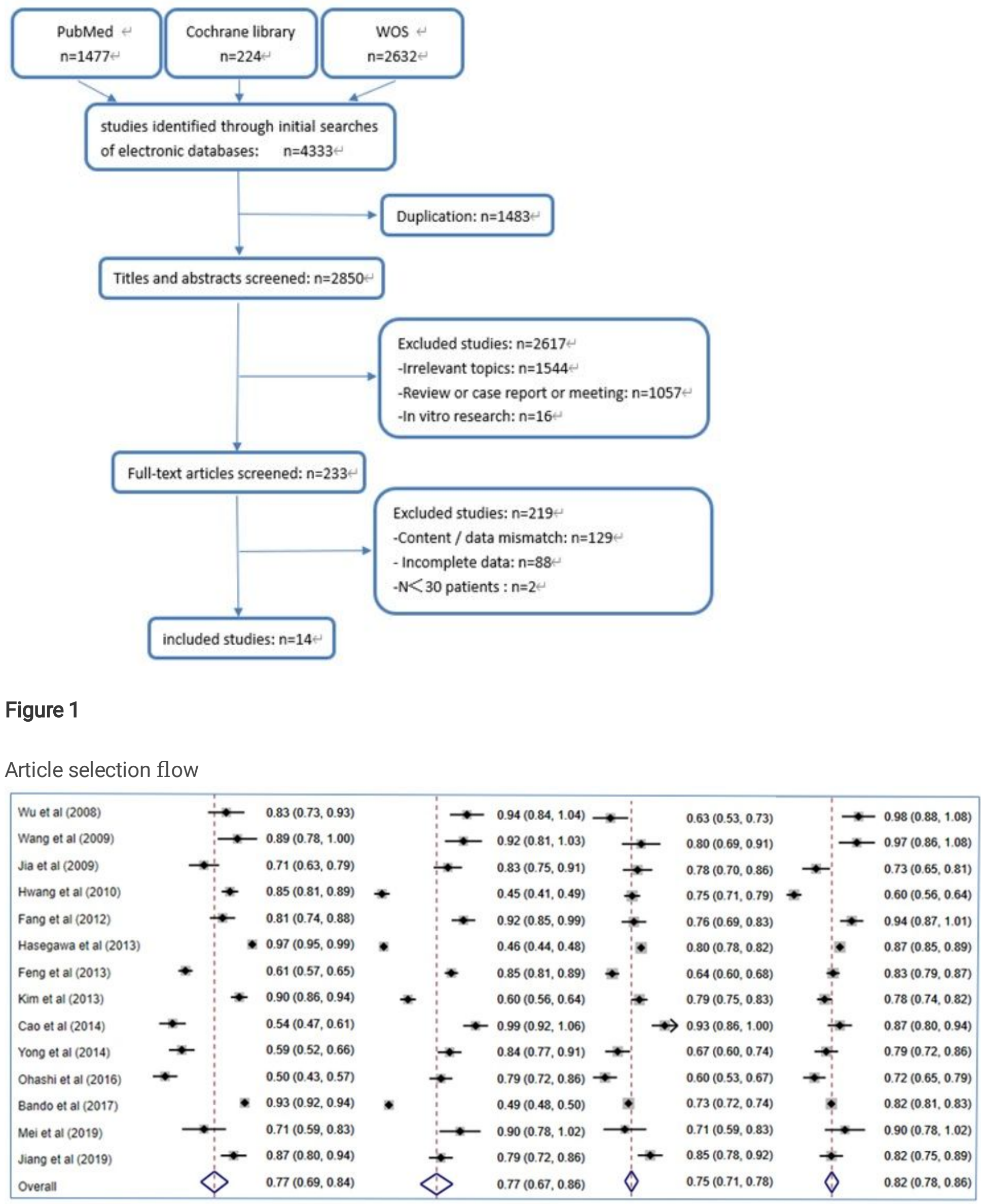

Figure 2 
MDCTsensitivity, specificity, PPV,NPV for NO staging

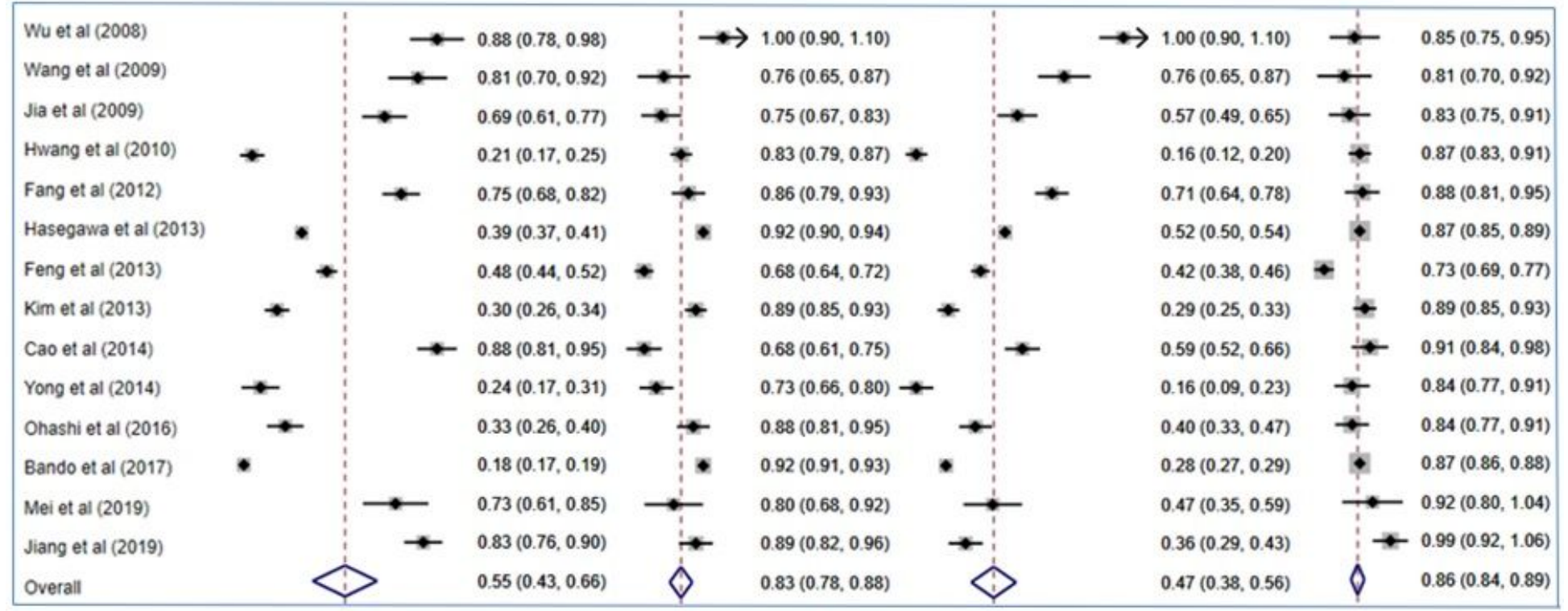

\section{Figure 3}

MDCT sensitivity, specificity, PPV, NPV for N1 staging

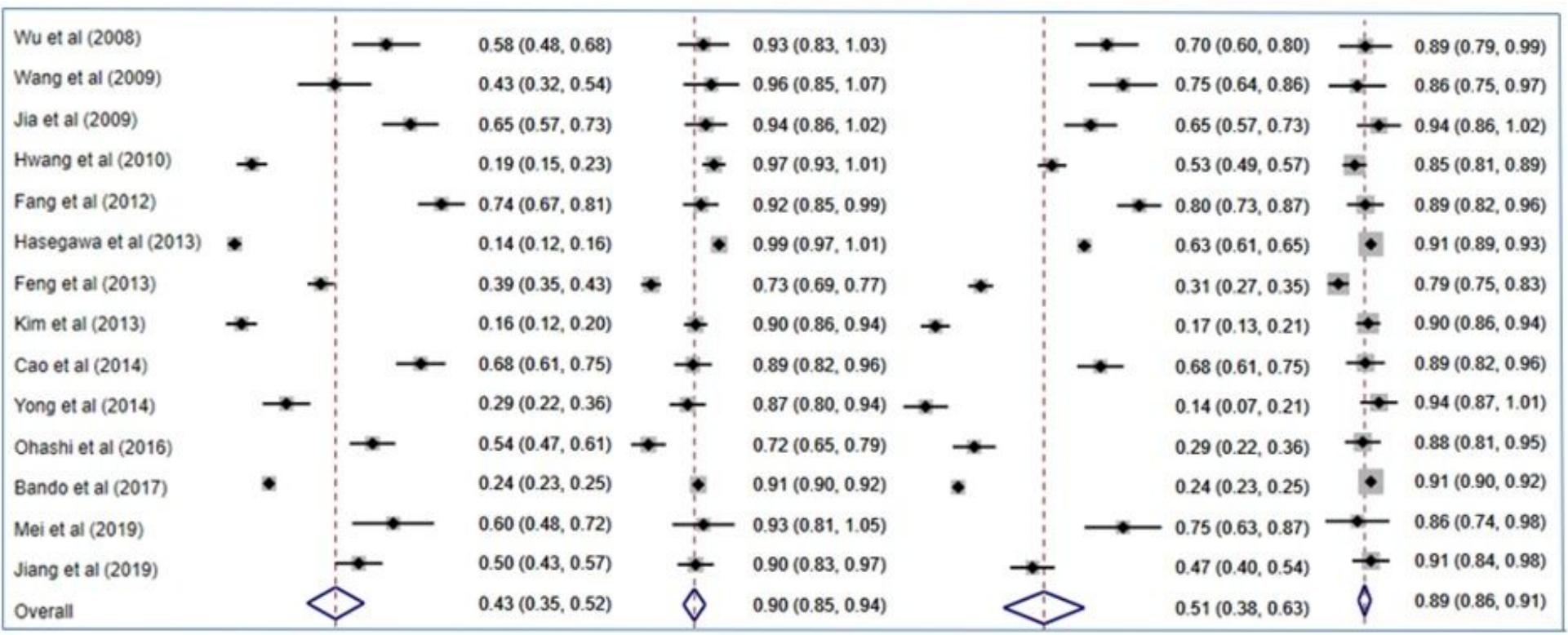

Figure 4

MDCT sensitivity, specificity, PPV, NPV for N2 staging 


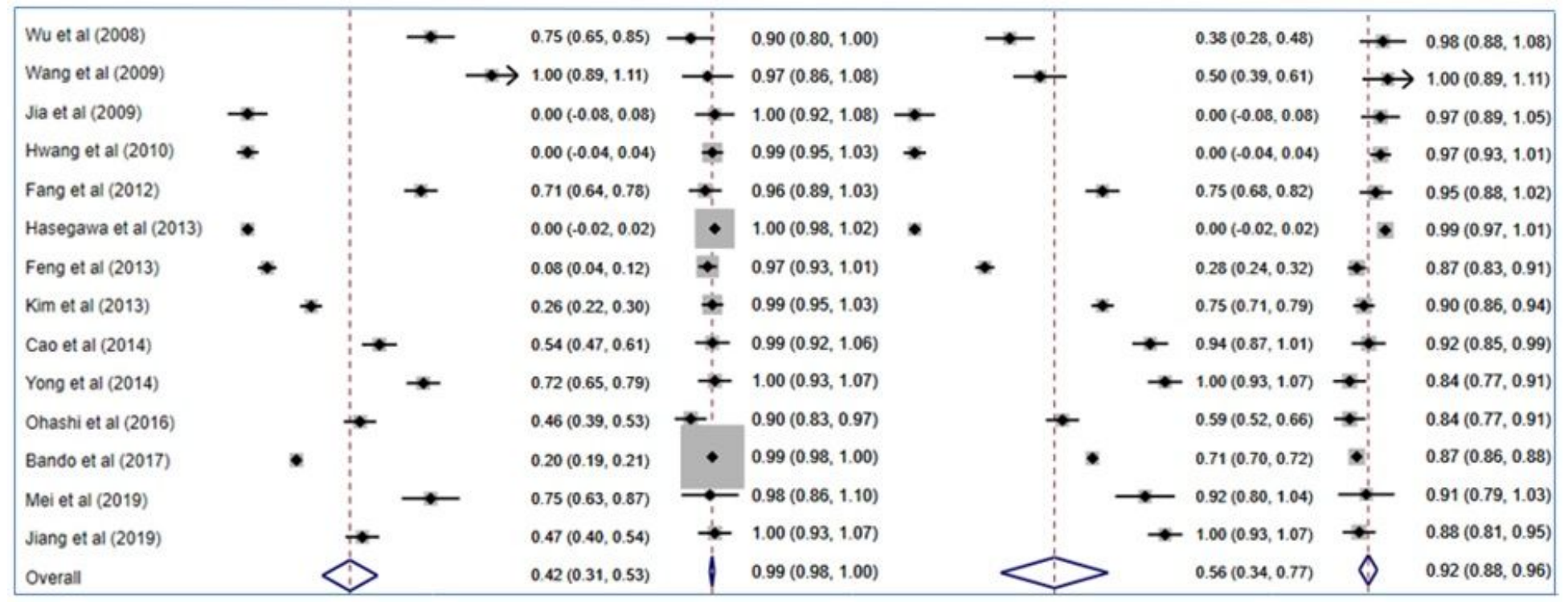

Figure 5

MDCT sensitivity, specificity, PPV, NPV for N3 staging

\section{Supplementary Files}

This is a list of supplementary files associated with this preprint. Click to download.

- PRISMAchecklist.docx 